\title{
On the singularities of the inverse to a meromorphic function of finite order
}

Walter Bergweiler and Alexandre Eremenko

Abstract. Our main result implies the following theorem: Let $f$ be a transcendental meromorphic function in the complex plane. If $f$ has finite order $\rho$, then every asymptotic value of $f$, except at most $2 \rho$ of them, is a limit point of critical values of $f$.

We give several applications of this theorem. For example we prove that if $f$ is a transcendental meromorphic function then $f^{\prime} f^{n}$ with $n \geq 1$ takes every finite non-zero value infinitely often. This proves a conjecture of Hayman. The proof makes use of the iteration theory of meromorphic functions.

\section{Introduction and main results.}

In this paper by meromorphic function we mean a transcendental meromorphic function in the complex plane $\mathbb{C}$, if the domain of definition is not explicitly specified. Let $f: \mathbb{C} \rightarrow \overline{\mathbb{C}}=\mathbb{C} \cup\{\infty\}$ be a meromorphic function. The inverse function $f^{-1}$ can be defined on a Riemann surface which is conformally equivalent to $\mathbb{C}$ via $f^{-1}$. In this paper we identify the Riemann surface of $f^{-1}$ with $\mathbb{C}$. We want to study the singularities of $f^{-1}$. This can be done by adding to $\mathbb{C}$ some 
ideal points and defining neighborhoods of these points.

Let us start with precise definitions. Take $a \in \overline{\mathbb{C}}$ and denote by $D(r, a)$ the disk of radius $r>0$ (in spherical metric) centered at $a$. For every $r>0$ choose a component $U(r)$ of the preimage $f^{-1}(D(r, a))$ in such a way that $r_{1}<r_{2}$ implies $U\left(r_{1}\right) \subset U\left(r_{2}\right)$. Note that the function $U: r \rightarrow U(r)$ is completely determined by its germ at 0 . Two possibilities can occur:

a) $\bigcap_{r>0} U(r)=\{z\}, z \in \mathbb{C}$. Then $a=f(z)$. If $a \in \mathbb{C}$ and $f^{\prime}(z) \neq 0$ or if $a=\infty$ and $z$ is a simple pole of $f$, then $z$ is called an ordinary point. If $a \in \mathbb{C}$ and $f^{\prime}(z)=0$ or if $a=\infty$ and $z$ is a multiple pole of $f$, then $z$ is called a critical point and $a$ is called a critical value. We also say that the critical point $z$ lies over $a$.

b) $\bigcap_{r>0} U(r)=\varnothing$. Then we say that our choice $r \rightarrow U(r)$ defines a (transcendental) singularity of $f^{-1}$. For simplicity we just call such $U$ a singularity. We also say that the singularity $U$ lies over $a$. For every $r>0$ the open set $U(r) \subset \mathbb{C}$ is called a neighborhood of the singularity $U$. So if $z_{k} \in \mathbb{C}$, we say that $z_{k} \rightarrow U$ if for every $\varepsilon>0$ there exists $k_{0}$ such that $z_{k} \in U(\varepsilon)$ for $k \geq k_{0}$.

If $U$ is a singularity then $a$ is an asymptotic value, which means that there exists a curve $\Gamma \subset \mathbb{C}$ tending to $\infty$ such that $f(z) \rightarrow a$ as $z \rightarrow \infty, z \in \Gamma$. Such $\Gamma$ is called an asymptotic curve. To construct an asymptotic curve take a sequence $r_{k} \rightarrow 0$ and a sequence $z_{k} \in U\left(r_{k}\right)$ and connect $z_{k}$ to $z_{k+1}$ by a curve $\gamma_{k} \subset U\left(r_{k}\right)$, which is possible because the $U(r)$ are connected. Then $\Gamma=\cup \gamma_{k}$ is an asymptotic curve. In particular it follows that every neighborhood $U(r)$ of a singularity $U$ is unbounded. If $a$ is an asymptotic value of $f$, then there is at least one singularity over $a$. Indeed, let $\Gamma \subset \mathbb{C}$ be an asymptotic curve, on which $f(z) \rightarrow a$. Then for every $r>0$ the "tail" of $\Gamma$ where $f(z) \in$ $D(r, a)$ belongs to $f^{-1}(D(r, a))$ and we define $U(r)$ as the component of $f^{-1}(D(r, a))$ which contains this tail.

Certainly there can be many different singularities as well as critical and ordinary points over the same point $a$. Remark that if $f$ is a meromorphic function, and $D \subset \overline{\mathbb{C}}$ contains no critical values and no asymptotic values then $f: f^{-1}(D) \rightarrow D$ is a covering. This justifies the name "singularities of $f^{-1}$ ".

The connection between asymptotic values of $f$ and singularities of $f^{-1}$ was stated for the first time by A. Hurwitz [17]. The following classification of singularities is due to F. Iversen [18] (see also [21], [26]). 
A singularity $U$ over $a$ is called direct if there exists $r>0$ such that $f(z) \neq a$ for $z \in U(r)$. (Then this is also true for all smaller values of $r$.) The simplest case of a direct singularity is the so-called logarithmic branch point. We say that $U$ is a logarithmic branch point (or logarithmic singularity) over $a$ if $f: U(r) \rightarrow D(r, a) \backslash\{a\}$ is a universal covering for some $r>0$. Thus if $f=\exp$ then the inverse function $f^{-1}=\log$ has two logarithmic branch points: one over 0 and one over $\infty$. The function arccos, inverse to cos, has two logarithmic singularities over $\infty$.

A singularity $U$ over $a$ is called indirect if it is not direct, i.e. for every $r>0$ the function $f$ takes the value $a$ in $U(r)$. In this case evidently the function $f$ takes the value $a$ infinitely often in $U(r)$. A simple example of an indirect singularity is given by the inverse function of $f(z)=\sin z / z$. Note that in this example the asymptotic value 0 is a limit point of critical values. M. Heins [15, Theorem 5] proved that the set of direct singularities of a function inverse to a meromorphic function is always countable.

For a meromorphic function of finite order $\rho$ the celebrated DenjoyCarleman-Ahlfors Theorem states that the inverse function has at most $\max \{2 \rho, 1\}$ direct singularities [21, p. 309]. This implies that an entire function of finite order $\rho$ has at most $2 \rho$ finite asymptotic values $[21, \mathrm{p}$. $313]$. On the other hand, there are meromorphic functions of any given order $\rho \geq 0$ such that every point in $\overline{\mathbb{C}}$ is an asymptotic value [7]. So in this case the number of indirect singularities is infinite.

In the simplest examples like $f(z)=\sin z / z$ the indirect singularities are limits of critical points. More complicated examples show that this is not the case in general. One such example is contained in the book of L. I. Volkovyskii [27, p. 70]. He constructs a meromorphic function $f$ with no critical points such that the set of asymptotic values has the power of the continuum (it is actually a Cantor set on the unit circle). So the inverse function of this function has many indirect singularities because the set of direct ones is countable by the result of Heins mentioned above. See also [25], where a similar example is discussed.

Our main result is that in the case of finite order the nature of the singularities of $f^{-1}$ is much simpler.

Theorem 1. If $f$ is a meromorphic function of finite order, then every indirect singularity of $f^{-1}$ is a limit of critical points. 
We can easily derive from Theorem 1 a formally stronger version of this theorem.

Theorem 1'. Let $f$ be a meromorphic function of finite order. Then every indirect singularity of $f^{-1}$ over $a \in \overline{\mathbb{C}}$ is a limit point of critical points $z_{k}$ such that $f\left(z_{k}\right) \neq a$.

Proof. Assume that $f$ has an indirect singularity $U$ over $a$ such that for some $r>0$ the set $V=U(r) \backslash f^{-1}(a)$ contains no critical points. As the number of direct singularities is finite we may assume that there are no direct singularities over $A=D(r, a) \backslash\{a\}$.

Let us show that

$$
f: V \rightarrow A
$$

has an asymptotic value $a^{\prime} \in A$. If this is not the case then (1) is a covering. As the fundamental group of the annulus $A$ is $\mathbb{Z}$ we conclude that the fundamental group of $V$ is $\mathbb{Z}$ or trivial. In the first case $V$ is a degenerate annulus and $a$ cannot be an asymptotic value in $U(r)$. So the fundamental group of $V$ is trivial, that is, (1) is a universal covering. Then $f: U(r) \rightarrow A$ is also a universal covering, which contradicts to our assumption that $U$ is a neighborhood of an indirect singularity over $a$.

Thus there is an asymptotic value $a^{\prime} \in A$ such that the corresponding (indirect) singularity $U^{\prime}$ has a neighborhood $U^{\prime}\left(r^{\prime}\right) \subset V$. Now we apply Theorem 1 to $U^{\prime}$ to conclude that there are critical points $z_{k} \in U(r)$ such that $f\left(z_{k}\right) \neq a$. This proves Theorem $1^{\prime}$.

Corollary 1. If $f$ is a meromorphic function of finite order and a is an asymptotic value of $f$, then $a$ is a limit of critical values $a_{k} \neq a$ or all singularities of $f^{-1}$ over a are logarithmic.

Corollary 2. If $f$ is a meromorphic function of finite order $\rho$ and $E$ is the set of its critical values, then the number of asymptotic values of $f$ is at most $2 \rho+$ card $E^{\prime}$, where $E^{\prime}$ stands for the derived set of $E$.

Proof. Let $a$ be an asymptotic value, $a \notin E^{\prime}$. By Corollary 1 there is a logarithmic singularity over $a$. Let us show that the number of logarithmic singularities is at most $2 \rho$. For $\rho \geq 1 / 2$ this follows from the Denjoy-Carleman-Ahlfors Theorem quoted above. It remains to show that there are no logarithmic singularities if $\rho<1 / 2$. Suppose 
that there is a logarithmic singularity over $a \in \overline{\mathbb{C}}$ and that $f: U(r) \rightarrow$ $D(r, a) \backslash\{a\}$ is a universal covering. Then $U(r)$ is a simply-connected unbounded domain. Assume without loss of generality that $a=\infty$. Then there exists $R>0$ such that $R<|f(z)|<+\infty$ for $z \in U(r)$ and $|f(z)|=R$ for $z \in \partial U(r)$. Define a function $u$ by $u(z)=\log (|f(z)| / R)$ for $z \in U(r)$ and $u(z)=0$ for $z \in \mathbb{C} \backslash U(r)$. It is easy to see that $u$ is subharmonic in $\mathbb{C}$. Since $u$ is bounded on $\partial U(r)$ we deduce from a classical theorem due to Wiman (see for example [14, Theorem 6.4]) that the order of $u$ is at least $1 / 2$. But the order of $f$ is greater or equal than the order of $u$.

Corollary 3. If a meromorphic function of finite order $\rho$ has only finitely many critical values, then it has at most $2 \rho$ asymptotic values.

Corollary 3 was conjectured by the second author in his talk on the A.M.S. meeting in Springfield, Missouri, in October 1991.

Theorem 1 and its corollaries may be useful in many questions involving meromorphic functions of finite order, in particular in the iteration theory of rational [1], [3], [24] and transcendental meromorphic [2] functions. The role of singularities in the iteration of transcendental functions is discussed in [2, Section 4.3]. The connection with rational functions is via Poincaré functions.

We will apply our result to the distribution of values of some differential polynomials. In [13, Problem 1.19] W. K. Hayman conjectured that if $f$ is a nonconstant meromorphic function and $n \in \mathbb{N}$, then $f^{\prime} f^{n}$ takes every finite non-zero value. Earlier he had proved this for $n \geq 3$. More precisely, he had shown that if $f$ is transcendental, then $f^{\prime} f^{n}$ takes every finite non-zero value infinitely often if $f$ is meromorphic and $n \geq 3$ [11, Corollary to Theorem 9] or if $f$ is entire and $n \geq 2[11$, Theorem 10]. J. Clunie [5] proved this for the case that $f$ is entire and $n=1$. Later E. Mues [19, Satz 3] settled the case that $f$ is meromorphic and $n=2$ and W. Hennekemper [16] extended Clunie's result to functions which have few poles in some sense.

We prove here the last unsolved case $(n=1$ for meromorphic functions). Our method gives also a unified proof of all results on Hayman's conjecture mentioned above.

Theorem 2. If $f$ is a transcendental meromorphic function and $m>l$ are positive integers then $\left(f^{m}\right)^{(l)}$ assumes every finite non-zero value infinitely often. 
Hayman's conjecture corresponds to the case $l=1$ in this theorem. The example $f(z)=e^{z}$ shows that 0 and $\infty$ can actually be omitted. Actually only the case $m=2, l=1$ in Theorem 2 is new. Recently Y. F. Wang [28] proved the statement of Theorem 2 for all $m \geq 3$ and all $l \geq 0$. Applying Theorem 2 to $1 / f$ instead of $f$ with $m=2$ and $l=1$ we obtain the following result.

Corollary 4. If $f$ is a transcendental meromorphic function then $f^{\prime}+$ $f^{3}$ has infinitely many zeros.

The corresponding result for $f^{\prime}+f^{n}, n \geq 4$, can be found in the papers of Hayman and Mues cited above.

Theorem 2 will be deduced from the following result which may be of independent interest.

Theorem 3. Let $f$ be a meromorphic function of finite order. If $f$ has infinitely many multiple zeros, then $f^{\prime}$ assumes every finite non-zero value infinitely often.

The proof of Theorem 3 uses iteration theory of meromorphic functions. The deduction of Theorem 2 from Theorem 3 is based on a rescaling lemma of Zalcman and Pang (Lemma 4), which allows to reduce the matter to the case of finite order. On the other hand we will construct an example which shows that Theorem 3 fails for functions of infinite order.

As a second application of Theorem 1 we give a unified proof of the following results recently obtained by J. Clunie, J. Langley, J. Rossi, and the second author [6], [8].

Theorem 4. Let $f$ be a transcendental meromorphic function of order $\rho$.

a) If $\rho<1$ then $f^{\prime}$ has infinitely many zeros.

b) If $\rho<1 / 2$ then $f^{\prime} / f$ has infinitely many zeros.

c) If $f$ is entire and $\rho<1$ then $f^{\prime} / f$ has infinitely many zeros.

Examples in [6] show that all bounds for $\rho$ in this theorem are sharp. 
REMARK. First we proved Hayman's conjecture (Theorem 2 with $m=$ 2 and $l=1$ ) only for functions of finite order. A preprint with this result was widely circulated. It was then realized independently (and almost simultaneously) by H. H. Chen and M. L. Fang, by L. Zalcman, and by the second author of this paper how the infinite order case can be reduced to the finite order case (Step 2 in the proof of Theorem 2). We are grateful to L. Yang for telling us about H. H. Chen's and M. L. Fang's result and to Y. F. Wang for sending us a preprint of their work [4], to L. Zalcman for informing us about his work and to D. Drasin for bringing to our attention the papers of X. Pang [22], [23].

\section{Lemmas.}

The proofs of the following two lemmas use some ideas of A. Weitsman [29] (compare also [8, Proposition 2.1]).

Lemma 1. Let $p>3$ be an integer and $g$ be a transcendental meromorphic function of order less than $p-3$. Then there exists an integer $n_{0}=n_{0}(g)$ and a sequence $R_{n} \in\left(2^{p n-2}, 2^{p n}\right), n \geq n_{0}$, such that the total length of the level curves $|g(z)|=R_{n}$ in $K_{n}=\left\{z:|z| \leq 2^{n}\right\}$ is at most $2^{\text {pn/2 }}$.

Proof. We use the standard notations of Nevanlinna theory [8], [12], [21]. For $R>|g(0)|+1$ (or $R>0$ if $g(0)=\infty$ ) and $\theta \in[0,2 \pi]$ we have

$n\left(2^{n}, \frac{1}{g-R e^{i \theta}}\right) \leq N\left(2^{n+2}, \frac{1}{g-R e^{i \theta}}\right) \leq T\left(2^{n+2}, g\right)+\log ^{+} R+C$

where $C$ depends on $g$ only. Thus

$$
\begin{aligned}
p_{n}(R) & :=\frac{1}{2 \pi} \int_{0}^{2 \pi} n\left(2^{n}, \frac{1}{g-R e^{i \theta}}\right) d \theta \\
& \leq T\left(2^{n+2}, g\right)+\log ^{+} R+C .
\end{aligned}
$$

Let $l_{n}(R)$ be the total length of the level curves $|g(z)|=R$ in $K_{n}$. Put $\beta_{n}=2^{p n}$ and $\alpha_{n}=2^{p n-2}$. By the length-area principle [10, p. 18] we have

$$
\int_{\alpha_{n}}^{\beta_{n}} \frac{l_{n}(R)^{2} d R}{R p_{n}(R)} \leq 2 \pi \text { area }\left(K_{n}\right)=2 \pi^{2} 2^{2 n} .
$$


So there exists $R_{n} \in\left(\alpha_{n}, \beta_{n}\right)$ such that

$$
l_{n}\left(R_{n}\right)^{2} \leq \frac{1}{\beta_{n}-\alpha_{n}} R_{n} p_{n}\left(R_{n}\right) 2 \pi^{2} 2^{2 n} \leq 2^{p n}, \quad n \geq n_{0},
$$

in view of (2) and the estimate

$$
T\left(2^{n+2}, g\right) \leq 2^{(p-3)(n+2)}, \quad n \geq n_{0} .
$$

This proves the lemma.

Lemma 2. Let $p>3$ be an integer and $f$ be a meromorphic function of order less than $p-3$. Given $\varepsilon>0$ there exists $C>0$ such that for every component $B$ of the set $E=\left\{z:\left|f^{\prime}(z)\right|<C^{-1}|z|^{-2 p}\right\}$ we have

$$
\operatorname{diam} f(B)<\varepsilon
$$

Here diam $S$ denotes the (Euclidean) diameter of a set $S \subset \mathbb{C}$.

Proof. Apply Lemma 1 to the function $g=1 / f^{\prime}$. Note that $f$ and $g$ have the same order because $f$ and $f^{\prime}$ have the same order by a result of J. M. Whittaker [30]. Increase if necessary $n_{0}$ from Lemma 1 such that

$$
\sum_{n=n_{0}}^{\infty} \frac{2^{n p / 2}+2 \pi 2^{n}}{R_{n}}<\frac{\varepsilon}{2}
$$

and hence

$$
\sum_{n=n_{0}}^{\infty} \frac{2^{n+1}}{R_{n}}<\frac{\varepsilon}{2} .
$$

For $n \geq n_{0}$ we set

$$
V_{n}=\left\{z:|z|<2^{n},|g(z)|>R_{n}\right\}
$$

and

$$
V=\bigcup_{n=n_{0}}^{\infty} V_{n}
$$


Note that the boundary $\partial V$ consists of some arcs of the level curves $|g(z)|=R_{n}$ which are in $K_{n}$ and some arcs of the circles $|z|=2^{n}$ on which we have $R_{n} \leq|g(z)| \leq R_{n+1}$. Applying Lemma 1 and (4) we obtain

$$
\int_{\partial V}|g(z)|^{-1}|d z| \leq \sum_{n=n_{0}}^{\infty} \frac{2^{n p / 2}+2 \pi 2^{n}}{R_{n}}<\frac{\varepsilon}{2} .
$$

We may assume without loss of generality that there are no poles of $g$ on $|z|=2^{n_{0}}$. Choose $C>1$ such that the set $E=\left\{z:|g(z)|>C|z|^{2 p}\right\}$ does not meet the circle $|z|=2^{n_{0}}$ and such that for all components $B$ of this set contained in $\left\{z:|z|<2^{n_{0}}\right\}$ the condition (3) is satisfied. Let us show that $E \cap\left\{z:|z| \geq 2^{n_{0}}\right\} \subset V$. If $z \in E$ and $|z| \geq 2^{n_{0}}$, we can find $n>n_{0}$ such that $2^{n-1} \leq|z|<2^{n}$. Then we have $|g(z)|>$ $C|z|^{2 p} \geq|z|^{2 p} \geq 2^{2 p(n-1)} \geq R_{n}$ so that $z \in V_{n} \subset V$.

Now let $D$ be a component of $V$ which contains a component $B$ of $E$ such that $B \subset\left\{z:|z|>2^{n_{0}}\right\}$. If $z_{1}$ and $z_{2}$ are in $B$, connect them by the straight line segment $L$. If $L \subset D$ take $\gamma=L$. If $L \not \subset D$ consider a segment $[a, b] \subset L$ such that $(a, b) \subset \mathbb{C} \backslash D$ and $a, b \in \partial D$. Replace $(a, b)$ by a bounded arc of $\partial D$ connecting $a$ and $b$. After performing this procedure on every segment of $L \backslash D$ we obtain a curve $\gamma_{1}$ connecting $z_{1}$ and $z_{2}$. Delete if necessary some parts of $\gamma_{1}$ to obtain a simple curve $\gamma$ connecting $z_{1}$ and $z_{2}$. The part of $\gamma$ in $D$ consists of some segments of $L$. Denote by $T_{n}$ the union of these segments which lie in $2^{n-1} \leq|z| \leq 2^{n}$. Then $|g(z)| \geq R_{n}$ for $z \in T_{n}$ and thus by (5) and (6)

$$
\begin{aligned}
\left|f\left(z_{1}\right)-f\left(z_{2}\right)\right| & \leq \int_{\gamma}|g(z)|^{-1}|d z| \\
& <\frac{\varepsilon}{2}+\sum_{n=n_{0}}^{\infty} \int_{T_{n}}|g(z)|^{-1}|d z| \\
& \leq \frac{\varepsilon}{2}+\sum_{n=n_{0}}^{\infty} \frac{2^{n+1}}{R_{n}} \\
& <\varepsilon .
\end{aligned}
$$




\section{Proof of Theorem 1.}

Let $a$ be an asymptotic value and $U$ be an indirect singularity over $a$ such that $U\left(R_{0}\right)$ contains no critical points and $0 \notin U\left(R_{0}\right)$ for some $R_{0}>0$. Without loss of generality we may assume that $a=0$. We are going to construct inductively the following objects:

- a sequence of asymptotic values $a_{n}, R_{0} / 2>\left|a_{1}\right|>\left|a_{2}\right|>\cdots$,

- a sequence of disjoint simply-connected domains $G_{n} \subset U\left(R_{0} / 2\right)$ such that $f$ is univalent in $G_{n}$ and $D_{n}=f\left(G_{n}\right)$ is a disk, $0 \notin \bar{D}_{n}$,

- a sequence of asymptotic curves $\Gamma_{n} \subset G_{n}$ such that $f\left(\Gamma_{n}\right)$ is a straight line segment and $f(z) \rightarrow a_{n}$ as $z \rightarrow \infty, z \in \Gamma_{n}$.

Let us show how to construct $a_{n}, G_{n}$, and $\Gamma_{n}$ assuming that $a_{k}$, $G_{k}$, and $\Gamma_{k}$ are already constructed for $k<n$.

First choose a positive number $R_{n}<\left|a_{n-1}\right|$ (if $n=1$ we take $\left.R_{1}<R_{0} / 2\right)$ such that $U\left(R_{n}\right) \cap G_{k}=\varnothing$ for $k<n$. This is possible because $0 \notin \bar{D}_{k}=\overline{f\left(G_{k}\right)}$. Then we take a point $z_{n} \in U\left(R_{n}\right)$ satisfying $f\left(z_{n}\right)=0$. The existence of such a point follows from the definition of an indirect singularity. We have $f^{\prime}\left(z_{n}\right) \neq 0$ by assumption. So there exists a branch $\varphi$ of $f^{-1}$ of the form

$$
\varphi(w)=z_{n}+\sum_{m=1}^{\infty} c_{m} w^{m} .
$$

Denote by $r_{n}$ the radius of convergence of this series.

We claim that

$$
0<r_{n}<R_{n}
$$

To prove the right inequality, suppose that $r_{n} \geq R_{n}$. Then $A=\varphi(\{w$ : $\left.\left.|w|<R_{n}\right\}\right)$ is a component of $f^{-1}\left(\left\{w:|w|<R_{n}\right\}\right)$, containing the point $z_{n} \in U\left(R_{n}\right)$. This implies that $A=U\left(R_{n}\right)$ because $U\left(R_{n}\right)$ is connected. Hence $f$ is univalent in $U\left(R_{n}\right)$, which is a contradiction. This proves $(7)$.

Let $a_{n}=r_{n} e^{i s_{n}}$ be a singular point of $\varphi$. We have $\left|a_{n}\right|=r_{n}<$ $R_{n}<\left|a_{n-1}\right|<\cdots<R_{0} / 2$.

Consider the disk

$$
D_{n}=\left\{w:\left|w-\frac{2 r_{n}}{3} e^{i s_{n}}\right|<\frac{r_{n}}{3}\right\} .
$$


Then $\varphi$ is holomorphic on $\bar{D}_{n} \backslash\left\{a_{n}\right\}$ and $0 \notin \bar{D}_{n}$. Set $G_{n}=\varphi\left(D_{n}\right)$. Then $G_{n}$ is a simply-connected domain in $\mathbb{C}$ bounded by one analytic curve tending to infinity in both directions. Indeed, if $G_{n}$ is bounded, then $z^{*}=\varphi\left(a_{n}\right) \in \mathbb{C}$. If $z^{*}$ is an ordinary point, then $\varphi$ has no singular point at $a_{n}$. But $z^{*} \in U\left(R_{n}\right) \subset U\left(r_{0}\right)$ cannot be a critical point by assumption. Moreover, $G_{n} \subset U\left(R_{n}\right)$ so that in particular $G_{n} \cap G_{k}=\varnothing$ for $k<n$. Finally we consider the segment

$$
L_{n}=\left\{w=t e^{i s_{n}}: \frac{2}{3} r_{n} \leq t<r_{n}\right\} \subset D_{n}
$$

and put $\Gamma_{n}=\varphi\left(L_{n}\right)$. This completes our construction.

Now we want to estimate the rate of convergence $f(z) \rightarrow a_{n}$, $z \in \Gamma_{n}$. Let $q_{n} \in \partial G_{n}, x_{n}=\left|q_{n}\right|$. For $x>x_{n}$ we denote by $\theta_{n}(x)$ the angular measure of $\left\{\theta: x e^{i \theta} \in G_{n}\right\}$. Then

$$
\sum_{n=1}^{\infty} \theta_{n}(x) \leq 2 \pi
$$

because the $G_{n}$ are disjoint. Now, by the Ahlfors distortion Theorem $[21$, p. 98$]$ applied to the conformal map $f: G_{n} \rightarrow D_{n}$, we have

$$
\log \frac{1}{\left|f(z)-a_{n}\right|} \geq \pi \int_{x_{n}}^{|z|} \frac{d x}{x \theta_{n}(x)}-C_{n}, \quad z \in \Gamma_{n},
$$

where the $C_{n}$ are constants. We want to conclude from here that for all $n$ with at most $4 p+2$ exceptions

$$
\liminf _{z \rightarrow \infty, z \in \Gamma_{n}}\left|f(z)-a_{n}\right||z|^{2 p+1}=0 .
$$

(Here $p>3$ is a natural number such that the order of $f$ is less than $p-3$.) To prove (10) assume that $\left|f(z)-a_{n}\right|>c|z|^{-2 p-1}$ for $K=4 p+3$ values of $n$ and all large $|z|$, say for $n=1,2, \ldots, K$ and $|z| \geq x_{0}$, where $x_{0}>\max \left\{x_{n}: 1 \leq n \leq K\right\}$. Then we have by (9)

$$
\pi \int_{x_{0}}^{|z|} \frac{d x}{x \theta_{n}(x)} \leq(2 p+1) \log |z|+O(1), \quad 1 \leq n \leq K .
$$

Now using Schwarz's inequality and (11) we get

$$
\left(\log \frac{|z|}{x_{0}}\right)^{2}=\left(\int_{x_{0}}^{|z|} \frac{d x}{x}\right)^{2} \leq
$$




$$
\begin{aligned}
& \leq \int_{x_{0}}^{|z|} \frac{d x}{x \theta_{n}(x)} \int_{x_{0}}^{|z|} \frac{\theta_{n}(x) d x}{x} \\
& \leq\left(\frac{1}{\pi}(2 p+1) \log |z|+O(1)\right) \int_{x_{0}}^{|z|} \frac{\theta_{n}(x) d x}{x} .
\end{aligned}
$$

Adding these inequalities for $n=1,2, \ldots, K$ and using (8) we obtain

$$
K\left(\log \frac{|z|}{x_{0}}\right)^{2} \leq(2(2 p+1) \log |z|+O(1)) \log \frac{|z|}{x_{0}}
$$

which is a contradiction because $K=4 p+3$. This proves that (10) is satisfied except possibly for $4 p+2$ values of $n$. Dropping those $a_{n}$ and $\Gamma_{n}$ for which (10) is not satisfied and changing the enumeration of the remaining $a_{n}$ and $\Gamma_{n}$ we may assume that (10) is satisfied for all $n$.

Next we prove that for every $n$ there exists a sequence $z_{n, j} \in \Gamma_{n}$, $z_{n, j} \rightarrow \infty$, such that

$$
\left|f^{\prime}\left(z_{n, j}\right)\right| \leq\left|z_{n, j}\right|^{-2 p-1}
$$

Recall that $f$ maps $\Gamma_{n}$ monotonically onto a straight line segment. Thus

$$
\left|f(z)-a_{n}\right|=\int_{z}^{\infty}\left|f^{\prime}(z)\right||d z|
$$

where the path of integration is $\Gamma_{n}$. If we assume contrary to (12) that $\left|f^{\prime}(z)\right|>|z|^{-2 p-1}$ for all $z \in \Gamma_{n}$ with sufficiently large moduli, then we obtain

$$
\left|f(z)-a_{n}\right| \geq \int_{z}^{\infty}|z|^{-2 p-1}|d z| \geq \frac{1}{2 p}|z|^{-2 p}
$$

which contradicts (10). Hence (12) is true.

Recall that $R_{0} / 2>\left|a_{1}\right|>\left|a_{2}\right|>\cdots$ and put

$$
\varepsilon=\frac{1}{4} \min \left\{\left|a_{i}-a_{j}\right|: 1 \leq i<j \leq 2 p\right\}
$$

Then $\varepsilon<R_{0} / 8$. Apply Lemma 2 using the value of $\varepsilon$ just specified. Lemma 2 gives some value $C>0$. For every $n$ choose a point $z_{n}^{*}=$ $z_{n, j(n)}$ using the relation (12) such that the following conditions are satisfied for $1 \leq n \leq 2 p$ :

$$
\left|z_{n}^{*}\right| \geq C
$$


and

$$
\left|f\left(z_{n}^{*}\right)-a_{n}\right|<\varepsilon
$$

Then

$$
\left|f\left(z_{n}^{*}\right)-f\left(z_{k}^{*}\right)\right|>2 \varepsilon, \quad 1 \leq n<k \leq 2 p,
$$

and

$$
\left|f\left(z_{n}^{*}\right)\right|+\varepsilon<\frac{3}{4} R_{0}, \quad 1 \leq n \leq 2 p .
$$

Using (12) and (13) we get

$$
\left|f^{\prime}\left(z_{n}^{*}\right)\right|<C^{-1}\left|z_{n}^{*}\right|^{-2 p}, \quad 1 \leq n \leq 2 p .
$$

Let $B_{n}$ be the component of the set $\left\{z:\left|f^{\prime}(z)\right|<C^{-1}|z|^{-2 p}\right\}$ containing $z_{n}^{*}$. Applying Lemma 2 we conclude that

$$
\operatorname{diam} f\left(B_{n}\right)<\varepsilon, \quad 1 \leq n \leq 2 p .
$$

By (15) we have $f\left(B_{n}\right) \subset\left\{w:|w|<3 R_{0} / 4\right\}$. But $U\left(R_{0}\right)$ is a component of $f^{-1}\left(\left\{w:|w|<R_{0}\right\}\right)$ and $U\left(R_{0}\right)$ and $B_{n}$ have a point $z_{n}^{*}$ in common. So we conclude that

$$
\bar{B}_{n} \subset U\left(R_{0}\right), \quad 1 \leq n \leq 2 p
$$

Comparing (14) and (17) we conclude that the $B_{n}$ are disjoint.

The function

$$
u(z)=-\log \left|f^{\prime}(z)\right|-2 p \log |z|-\log C
$$

is subharmonic in $U\left(R_{0}\right)$ because $U\left(R_{0}\right)$ does not contain critical points of $f$ by assumption. Also $0 \notin U\left(R_{0}\right)$ by assumption. Now the $B_{n}$ are components of the set $\left\{z \in U\left(R_{0}\right): u(z)>0\right\}$ and we have $u(z)=0$ for $z \in \partial B_{n}$ by (18).

Now a standard application of the subharmonic version of the Denjoy-Carleman-Ahlfors Theorem [14, Theorem 8.9] shows that the order of $u$ is at least $p$. So the order of $f^{\prime}$ and hence $f$ is at least $p$ and we have a contradiction which proves the theorem. 


\section{Proof of Theorems 2 and 3.}

Proof of Theorem 3. Let $c \in \mathbb{C} \backslash\{0\}$ and consider the function $g$ defined by $g(z)=z-f(z) / c$. Then $g$ has finite order because $f$ has finite order.

We shall use some results from the iteration theory of meromorphic functions. By $g^{\circ n}$ we denote the $n$-th iterate of $g$. The largest open set where all $g^{\text {on }}$ are defined and form a normal family is called the Fatou set of $g$ and denoted by $F(g)$.

Let now $\zeta$ be a multiple zero of $f$. Then $g(\zeta)=\zeta$ and $g^{\prime}(\zeta)=1$. Classical results from iteration theory (see for example [1, Theorem 6.5.4]) now imply that there exists a component $U$ of $F(g)$, a so-called Leau domain, such that $\zeta \in \partial U$ and $g^{\circ n} \rightarrow \zeta$ locally uniformly in $U$. Moreover, $U$ contains a critical or asymptotic value of $g$, see for example [1, Theorem 9.3.2]. (In [1] as well as in [3], [24] only the case of rational functions is discussed, in which case only critical values need to be considered, but the proof extends to the transcendental case, if we also take asymptotic values into account.) Since $f$ has infinitely many multiple zeros and since Leau domains related to distinct fixed points of $g$ are disjoint, we deduce that the set of critical and asymptotic values of $g$ is infinite. By Corollary 3 this is possible only if $g$ has infinitely many critical values. In particular, $g^{\prime}$ has infinitely many zeros which implies that $f^{\prime}$ assumes the value $c$ infinitely often. This completes the proof of Theorem 3.

For the proof of Theorem 2 we also need the following lemmas.

Lemma 3. Let $f$ be a transcendental meromorphic function. If $f$ has only finitely many zeros, then $f^{(l)}, l \geq 1$, assumes every finite non-zero value infinitely often.

Lemma 3 was proved by W. K. Hayman ([11, Theorem 3] or [12, Corollary to Theorem 3.5]).

Lemma 4. Let $F$ be a non normal family of meromorphic functions in the unit disk $D$, and $-1<k<1$. Then there exist sequences $f_{n} \in F$, $z_{n} \in D$ and $a_{n}>0$ such that $\left|z_{n}\right|<r<1, a_{n} \rightarrow 0$ and

$$
g_{n}(\zeta)=a_{n}^{-k} f_{n}\left(z_{n}+a_{n} \zeta\right) \rightarrow g(\zeta),
$$

where $g$ is a non-constant meromorphic function in the plane of order at most 2, normal type, and the convergence is uniform on compacta in $\mathbb{C}$ with respect to the spherical metric. 
The case $k=0$ in Lemma 4 was proved by L. Zalcman [31], [32], and the general case by X. Pang [22], [23].

\section{Proof of Theorem 2.}

Step 1. We first prove the theorem for the case when the order of $f$ is finite. If $f$ has finitely many zeros then the conclusion follows from Lemma 3. If $f$ has infinitely many zeros then $h=\left(f^{m}\right)^{(l-1)}$ has infinitely many multiple zeros and we apply Theorem 3 to $h$.

Step 2. Now we reduce the general case to the case of finite order, using Lemma 4. We use the notation

$$
f^{\#}=\frac{\left|f^{\prime}\right|}{1+|f|^{2}}
$$

for the spherical derivative.

Suppose that there exists a transcendental meromorphic function $f$ such that the equation $\left(f^{m}\right)^{(l)}(z)=a$ has a finite set of solutions for some $a \neq 0$. We may assume without loss of generality that $a=1$.

Put $k=l / m$ and define a family $F$ consisting of all functions

$$
f_{n}(z)=2^{-k n} f\left(2^{n} z\right), \quad 1 / 4<|z|<2, \quad n=1,2, \ldots
$$

This family cannot be normal in $\{z: 1 / 4<|z|<2\}$. For otherwise we would have for some $M>0$

$$
M>f_{n}^{\#}(z) \geq 2^{(1-k) n} f^{\#}\left(2^{n} z\right)>f^{\#}\left(2^{n} z\right), \quad 1 / 2<|z|<1
$$

from which follows that

$$
\iint_{|x+i y|<r}\left(f^{\#}(x+i y)\right)^{2} d x d y=O\left(r^{2}\right), \quad r \rightarrow+\infty,
$$

so the order of $f$ is finite which contradicts Step 1.

Now notice that $\left(f_{n}^{m}\right)^{(l)}(z)=\left(f^{m}\right)^{(l)}\left(2^{n} z\right)$, so $\left(h^{m}\right)^{(l)}(z) \neq 1$ for every $h \in F$.

Now we choose a disk in the annulus $\{z: 1 / 4<|z|<2\}$ such that $F$ is not normal in this disk, apply Lemma 4 to $F$ with $k=l / m$ and obtain a non-constant meromorphic function $g$ of order at most 2 which also has the property $\left(g^{m}\right)^{(l)}(z) \neq 1, z \in \mathbb{C}$. This contradicts Step 1 . So Theorem 2 is proved. 
Here is another application of Theorem 3.

If $P$ is a non-constant polynomial and if $f$ is a transcendental meromorphic function of finite order, then $P(f) f^{\prime}$ assumes every finite non-zero value infinitely often. This was proved by E. Mues [20, Satz 1] for the case that $f$ is entire, but without the restriction on the order. To see this we choose a zero $a$ of $P$, with the property that $f$ has infinitely many $a$-points if such a zero exists. We define $Q(z)=\int_{a}^{z} P(t) d t$ and proceed as in the proof of Theorem 2, Step 1, with $h=Q(f)$.

Now we will show that Theorem 3 fails for functions of infinite order.

ExAmple. Define

$$
f(z)=z+a \int_{0}^{z} \exp (b \exp t-t) d t
$$

where $a$ and $b$ are complex numbers with the properties:

$$
1+a b=0 \quad \text { and } \quad 1+a \exp b=0 .
$$

Such numbers are easy to find by taking any solution of $\exp (z)=z$ as $b$ and putting $a=-1 / b$. From the first condition (19) follows that $f(2 \pi i)=0$. (Use the substitution $w=\exp t$ and residues to evaluate the integral). So $f$ has period $2 \pi i$. From the second condition (19) follows that $f^{\prime}(0)=0$. By periodicity $f$ has multiple zeros at the points $2 \pi i k$. On the other hand $f^{\prime}$ omits the value 1 .

\section{Proof of Theorem 4.}

We start with the following simple

Proposition Let $f$ be a meromorphic function with infinitely many zeros and no asymptotic values in $\mathbb{C}^{*}=\mathbb{C} \backslash\{0\}$. Then there are infinitely many critical points lying over $\mathbb{C}^{*}$.

ProOF. We have infinitely many branches of $f^{-1}$ of the form

$$
q_{k}(w)=\varphi_{k}\left(w^{1 / p_{k}}\right)=z_{k}+\sum_{n=1}^{\infty} c_{n} w^{n / p_{k}}
$$


where $p_{k}$ are some integers, $z_{k}$ are the zeros of $f$ and $\varphi_{k}$ are univalent. If the radius of convergence $r_{k}$ of the series $\varphi_{k}$ is infinite then $\varphi_{k}$ is linear as a univalent holomorphic function in $\mathbb{C}$. So all $r_{k}$ are finite which means that the branches $q_{k}$ have singularities in $\mathbb{C}^{*}$. These singularities are algebraic branch points and only finitely many $q_{k}$ 's may share one such singularity. Thus the total number of critical points of $f$ over $\mathbb{C}^{*}$ is infinite. This completes the proof of the Proposition.

We will also use a theorem of F. Iversen [18], [21], which states that if a transcendental meromorphic function takes some value $a \in \overline{\mathbb{C}}$ finitely many times then $a$ is an asymptotic value.

Proof of Theorem 4. To prove a) assume that $f^{\prime}$ has finitely many zeros. Then all but a finite set of critical points lie over $\infty$. From Corollary 2 we conclude that there is at most one asymptotic value $a \in$ $\overline{\mathbb{C}}$. If $a=\infty$ or there is no asymptotic value at all, then $f$ has infinitely many zeros and we apply the Proposition to get a contradiction. If $a$ is finite we may assume without loss of generality that $a=0$. Then $f$ has infinitely many poles by Iversen's theorem and we apply the Proposition to $1 / f$.

To prove b) we assume that $f^{\prime} / f$ has finitely many zeros. This means that all except finitely many critical points lie over 0 and $\infty$. By Corollary 2 there are no asymptotic values. So we have infinitely many zeros by Iversen's theorem and the Proposition gives a contradiction.

To prove c) we assume that $f^{\prime} / f$ has finitely many zeros. Then all critical values lie over 0 and by Corollary 2 and Iversen's theorem the only asymptotic value is infinity. Again there are infinitely many zeros and the application of the Proposition finishes the proof.

\section{References.}

[1] Beardon, A. F., Iteration of Rational Functions. Springer-Verlag, 1991.

[2] Bergweiler, W., Iteration of meromorphic functions, Bull. Amer. Math. Soc. 29 (1993), 151-188.

[3] Carleson, L., Gamelin, T. W., Complex Dynamics, Springer-Verlag, 1993.

[4] Chen, H. H., Fang, M. L., On the value distribution of $f^{n} f^{\prime}$. Preprint.

[5] Clunie, J., On a result of Hayman. J. London Math. Soc. 42 (1967), 389-392. 
[6] Clunie, J., Eremenko, A. and Rossi, J., On equilibrium points of logarithmic and Newtonian potentials, J. London Math. Soc. 47 (1993), 309-320.

[7] Eremenko, A., The set of asymptotic values of a meromorphic function of finite order, Math. Notes 24 (1979), 914-916.

[8] Eremenko, A., Langley, J. and Rossi, J., On the zeros of meromorphic functions of the form $f(z)=\sum_{k=1}^{\infty} a_{k} /\left(z-z_{k}\right)$. J. Analyse Math., to appear.

[9] Gol'dberg, A. A. and Ostrovski, I. V., Distribution of Values of Meromorphic Functions (Russian). Nauka, 1970.

[10] Hayman, W. K., Multivalent Functions. Cambridge, 1958.

[11] Hayman, W. K., Picard values of meromorphic functions and their derivatives. Ann. of Math. 70 (1959), 9-42.

[12] Hayman, W. K., Meromorphic Functions. Oxford at the Clarendon Press, 1964.

[13] Hayman, W. K., Research Problems in Function Theory. The Athlone Press, 1967.

[14] Hayman, W. K., Subharmonic Functions, Vol. 2. Academic Press, 1989.

[15] Heins, M., Asymptotic spots of entire and meromorphic functions. Ann. of Math. 66 (1957), 430-439.

[16] Hennekemper, W., Über die Werteverteilung von $\left(f^{k+1}\right)^{(k)}$. Math. Z. 177 (1981), 375-380.

[17] Hurwitz, A., Sur les points critiques des fonctions inverses. Comptes Rendus 143 (1906), 877-879; Math. Werke, Bd. 1, S. 655-656.

[18] Iversen, F., Recherches sur les fonctions inverses des fonctions méromorphes. Thèse, Helsingfors, 1914.

[19] Mues, E., Über ein Problem von Hayman. Math. Z. 164 (1979), 239259.

[20] Mues, E., Zur Werteverteilung von Differentialpolynomen. Arch. Math. 32 (1979), 55-67.

[21] Nevanlinna, R., Eindeutige analytische Funktionen. Springer-Verlag, 1953.

[22] Pang, X., Bloch's principle and normal criterion. Science in China 32 (1989), 782-791.

[23] Pang, X., On normal criterion of meromorphic functions. Science in China 33 (1990), 521-527.

[24] Steinmetz, N., Rational Iteration, Walter de Gruyter, 1993.

[25] Stephenson, K., Concerning the Gross Star Theorem. Complex Analysis, Joensuu 1987, 328-338, Lecture Notes in Math., Springer-Verlag, 1988.

[26] Tsuji, M., Potential Theory in Modern Function Theory. Maruzen, 1959. 
[27] Volkovyskii, L. I., Research on the Type Problem of a Simply Connected Riemann Surface. Proc. Steklov Math. Inst., Acad. Sci. USSR. 34, 1950.

[28] Wang, Y. F., On Mues conjecture and Picard values. Preprint, Inst. Math. Acad. Sinica.

[29] Weitsman, A., A theorem on Nevanlinna deficiencies. Acta Math. 128 (1972), 41-52.

[30] Whittaker, J. M., The order of the derivative of a meromorphic function. J. London Math. Soc. 11 (1936), 82-87.

[31] Zalcman, L., A heuristic principle in complex function theory. Amer. Math. Monthly 82 (1975), 813-817.

[32] Zalcman, L., Normal families revisited, in Complex Analysis and related topics, J. Wiegerinck, ed., Univ. of Amsterdam, 1993, 149-164.

Recibido: 24 de septiembre de 1.993

Revisado: 11 de agosto de 1.994

Walter Bergweiler

Fachbereich Mathematik

Sekr. MA 8-2, TU-Berlin

Straße des 17. Juni, 10623 Berlin, GERMANY

bergweil@math.tu-berlin.de

and

Alexandre Eremenko*

Department of Mathematics

Purdue University

West Lafayette, IN 47907, U.S.A.

eremenko@math. purdue . edu

\footnotetext{
* Sponsored by the Graduiertenkolleg "Analyse und Konstruktion in der Mathematik" at the Technical University of Aachen when this paper was written. He also thanks NSF for partial support.
} 